\title{
THE PHOTOSTABILITY OF AMINO ACIDS IN SPACE
}

\author{
P. Ehrenfreund, ${ }^{1}$ M. P. Bernstein, ${ }^{2,3}$ J. P. Dworkin, ${ }^{2,3}$ S. A. Sandford, ${ }^{2}$ And L. J. Allamandola ${ }^{2}$ \\ Received 2000 December 12; accepted 2001 January 31; published 2001 March 13
}

\begin{abstract}
Organic compounds observed in the interstellar medium and in solar system bodies are of particular importance for revealing the chemistry that may have led to life's origin. Among these compounds, amino acids may have played a crucial role since they are basic components of proteins, which are the essential constituents of all organisms. We present laboratory studies testing the stability of amino acids against ultraviolet (UV) photolysis. Two biological and two nonbiological amino acids have been irradiated in frozen $\mathrm{Ar}, \mathrm{N}_{2}$, and $\mathrm{H}_{2} \mathrm{O}$ to simulate conditions in the interstellar gas and on interstellar grains. The experimental results can be interpreted to indicate that amino acids in the gas phase will likely be destroyed during the lifetime of a typical interstellar cloud. In regions with relatively low UV radiation, amino acids might be present as transient gas-phase species. Their survival in interstellar icy grain mantles and the surface layers of comets and planets is strongly limited in the presence of UV irradiation. The rate of destruction is rather insensitive to the amino acid structure and to the ice matrix. We consider the implications of these results for the survival and transfer of amino acids in space environments, and thus their possible availability for prebiotic chemistry.
\end{abstract}

Subject headings: infrared: ISM: lines and bands — ISM: abundances — ISM: molecules methods: laboratory - molecular processes

\section{INTRODUCTION}

Extraterrestrial prebiotic molecules, including aldehydes, ethers, quinones, and amino acids, may have played an important role in the origin and evolution of life (Oró 1961; Chyba et al. 1990; Bernstein, Sandford, \& Allamandola 1999). Amino acids have been identified in several carbonaceous meteorites in concentrations of up to 3 parts per million versus carbon (Cronin \& Chang 1993). Whereas life on Earth is based on Lamino acids (left-handed), those measured in meteorites are $50 \%$ in D-form (right-handed) or racemic (mixed handedness). Small excesses of left-handed extraterrestrial amino acids, such as isovaline, have been reported in the Murchison and Murray meteorites (Cronin \& Pizzarello 1997).

The fact that meteoritic amino acids are deuterium-enriched (Pizzarello et al. 1991; Sandford 1996; Irvine 1998) may imply an interstellar heritage. Formation of amino acids in the interstellar medium (ISM) may be possible via specific gas-phase reactions in dark clouds (Ehrenfreund \& Charnley 2000). At present, the detection of glycine $\left(\mathrm{H}_{2} \mathrm{NCH}_{2} \mathrm{COOH}\right.$; the simplest amino acid) in the interstellar gas remains controversial (Snyder 1997; Charnley, Ehrenfreund, \& Kuan 2001); an upper limit of $10^{-10}$ (per $\mathrm{H}_{2}$ ) has recently been estimated in the low-mass protostar IRAS 16293-2422 (Ceccarelli et al. 2000).

To assess the availability of amino acids for prebiotic chemistry, it is imperative to investigate the stability of such compounds in extraterrestrial environments. Whereas recent studies focused on the thermal properties of amino acids and their stability upon impact (Pierazzo \& Chyba 1999; Blank, Miller, \& Winans 2001), we tested the photostability of four amino acids in $\mathrm{Ar}, \mathrm{N}_{2}$, and $\mathrm{H}_{2} \mathrm{O}$ ices to explore whether they could withstand radiation in space. Whereas glycine $\left(\mathrm{NH}_{2} \mathrm{CH}_{2} \mathrm{COOH}\right)$ and L-alanine $\left[\mathrm{NH}_{2} \mathrm{CH}\left(\mathrm{CH}_{3}\right) \mathrm{COOH}\right]$ are common in living organisms, $\alpha$-aminoisobutyric acid $\left[\mathrm{NH}_{2} \mathrm{C}\left(\mathrm{CH}_{3}\right)_{2} \mathrm{COOH}\right]$ and $\beta$ -

\footnotetext{
${ }^{1}$ Sackler Laboratory for Astrophysics, Leiden Observatory, P.O. Box 9513, 2300 RA Leiden, Netherlands; pascale@strw.leidenuniv.nl.

${ }^{2}$ Space Science Division, NASA Ames Research Center, MS 245-6, Moffett Field, CA 94305-1000.

${ }^{3}$ SETI Institute, 2035 Landings Drive, Mountain View, CA 94043.
}

alanine $\left(\mathrm{NH}_{2} \mathrm{CH}_{2} \mathrm{CH}_{2} \mathrm{COOH}\right)$ are extremely rare on Earth. In $\alpha$-amino acids, the amino group $\left(-\mathrm{NH}_{2}\right)$ is attached to the $\mathrm{C}$ atom positioned next to the carboxy group $(-\mathrm{COOH})$, whereas in $\beta$-amino acids, the amino group is attached to a $\mathrm{C}$ atom that is two carbon-carbon bonds distant from the carboxy group. The sequence to test glycine, L-alanine, $\beta$-alanine, and $\alpha$ aminoisobutyric acid (AIB) was chosen in order to investigate how the size and structure of amino acids would influence their destruction by UV photons. While we have restricted our studies to UV radiation, the general conclusions may apply to cosmic rays as well (Gerakines, Moore, \& Hudson 2000).

\section{EXPERIMENTAL}

The spectra and photochemistry of amino acid samples were carried out using standard matrix isolation techniques that are described elsewhere (Hudgins \& Allamandola 1995). For the matrix materials, we employed $99.999 \%$ pure $\mathrm{Ar}$ or $\mathrm{N}_{2}$ (Matheson) and triply freeze-pump-thawed $18 \mathrm{M} \Omega \mathrm{H}_{2} \mathrm{O}$ (Millipore). The amino acids glycine, D, L-alanine, $\alpha$-aminoisobutyric-acid, and $\beta$-alanine were purchased from Sigma (purity of $99 \%$ ). The amino acid samples were sublimed from a borosilicate tube at about $150^{\circ} \mathrm{C}$ onto a $12 \mathrm{~K} \mathrm{CsI}$ window along with the matrix gas flowing from a separate inlet port. The Ar or $\mathrm{N}_{2}$ matrix thickness was estimated from infrared (IR) interference fringes (from internal reflections within the sample layer), while the amount of $\mathrm{H}_{2} \mathrm{O}$ ice was determined from the 3,6 , and $15 \mu \mathrm{m}$ band areas. The amount of amino acids was estimated from the area of the amino acid carbonyl $(\mathrm{C}=\mathrm{O})$ stretching mode near $1780 \mathrm{~cm}^{-1}$, assumed to have a cross section of $\sim 3 \times 10^{-17} \mathrm{~cm}$ molecule ${ }^{-1}$ (Wexler 1967). Typically, the $\mathrm{C}=\mathrm{O}$ stretching feature had a depth of $\sim 10 \%$ after a few minutes of deposition, while the thickness varied from $1 \mu \mathrm{m}$ for the Ar matrix to less than $0.2 \mu \mathrm{m}$ for $\mathrm{N}_{2}$ and $\mathrm{H}_{2} \mathrm{O}$ ices (in order to guarantee full UV penetration). Based on these estimates, we determined that the amino acids were isolated in the Ar matrix (matrix gas/amino acid or $M / R>300: 1$ ) and that approximately $M / R=10: 1$ in $\mathrm{N}_{2}$ and $\mathrm{H}_{2} \mathrm{O}$ ices.

UV irradiation was performed using a microwave-excited hydrogen flow lamp, described in detail elsewhere (Warneck 

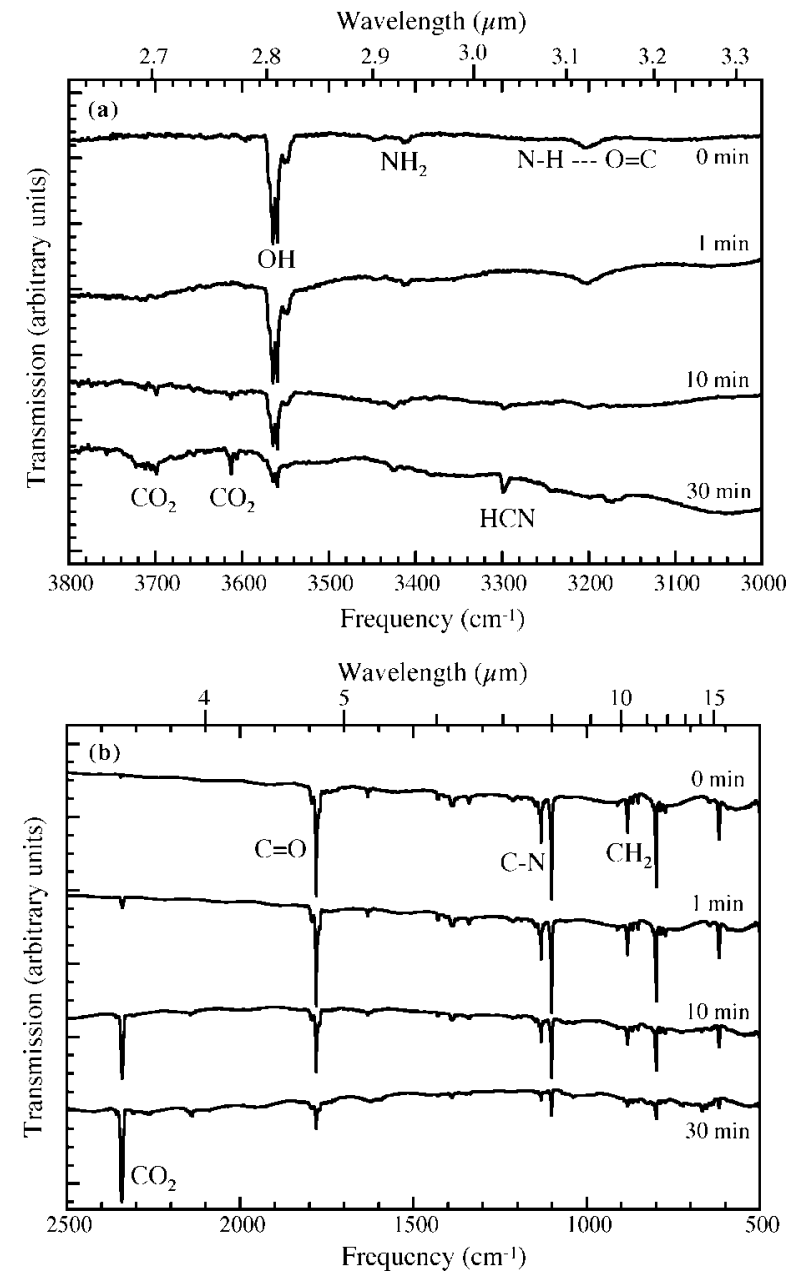

FIG. 1.-IR spectrum of glycine isolated in $\mathrm{Ar}$ at $12 \mathrm{~K}$ between $(a)$ $3800-3000$ and (b) $2500-500 \mathrm{~cm}^{-1}$. The bands associated with the main functional groups on the glycine starting material are labeled on the top trace. The dilution factor, calculated from the Ar matrix fringes and band area of the carbonyl $\mathrm{C}=\mathrm{O}$ amino acid stretch (see text), is greater than 300:1 ( $\mathrm{Ar}$ : glycine). Upon exposure to UV radiation for 1,10 , and 30 minutes, all bands due to glycine diminish simultaneously. No IR signature of glycine remains after $1 \mathrm{hr}$ of exposure to UV irradiation. Simultaneous with the disappearance of the amino acid bands is the appearance of new bands attributed to photoproducts, some of which are identified in the spectrum. The IR transitions of amino acids clearly visible in an Ar matrix are masked when those compounds are embedded in $\mathrm{H}_{2} \mathrm{O}$ ice. It is therefore highly unlikely that amino acids can be detected by IR spectroscopy in astrophysical environments that are dominated by $\mathrm{H}_{2} \mathrm{O}$ ice.

1962). Half-lives for the photodecomposition of amino acids in $\mathrm{Ar}$ and $\mathrm{N}_{2}$ were determined by a first-order fit to a plot of the disappearance of the amino acid carbonyl stretch with time, as seen in Figure 2 below. The equation used for the fit was an exponential of the form $a e^{-b t}$, where $e$ is the natural $\log$ base, $t$ is the time in seconds, and the variable $b$ is the rate constant for amino acid destruction in inverse seconds. The half-life is the amount of time required for $50 \%$ of the starting material to decompose. Since the amino acid carbonyl stretch is obscured in $\mathrm{H}_{2} \mathrm{O}$ ice, the half-life for the photodecomposition of amino acids in $\mathrm{H}_{2} \mathrm{O}$ was estimated from the formation of $\mathrm{CO}_{2}$. While this is valid in the Ar matrix, it may lead to small systematic errors in the determination of half-lives in $\mathrm{H}_{2} \mathrm{O}$ ice since the acid group on the amino acid is not the only source of $\mathrm{CO}_{2}$. Following in situ IR spectroscopy, samples were warmed to $298 \mathrm{~K}$ at $\sim 1 \mathrm{~K}$ per minute under a dynamic vacuum.

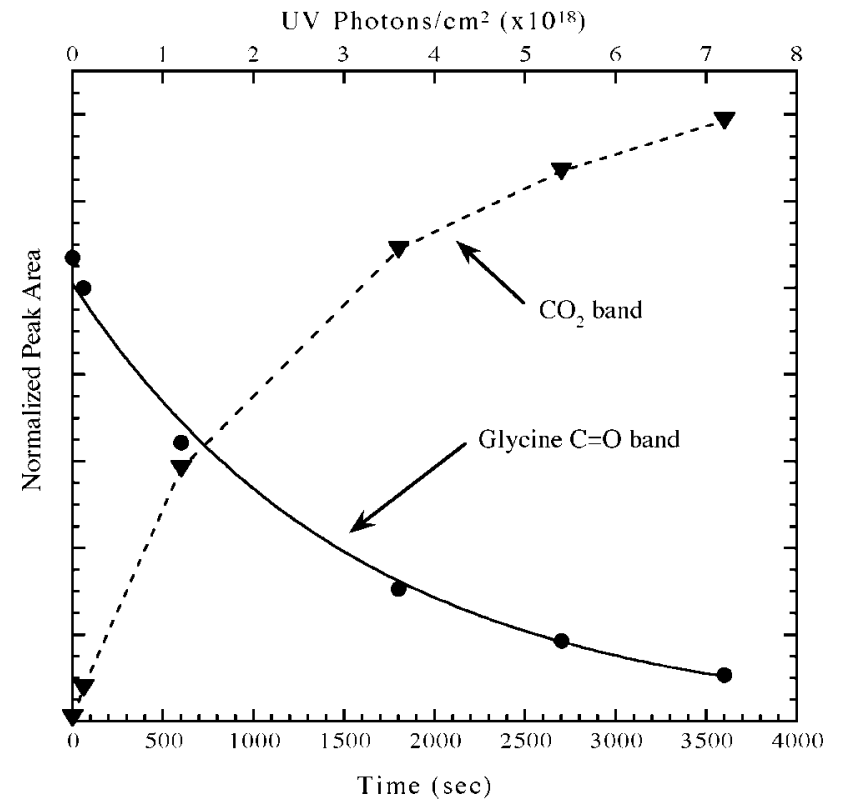

FIG. 2.-Diminishing area of the carbonyl $(\mathrm{C}=\mathrm{O})$ stretch of glycine (solid line) upon photolysis in solid $\mathrm{Ar}$ and the concomitant increase in the area of the asymmetric stretch of the photoproduct, $\mathrm{CO}_{2}$ (dashed line). This strong inverse correlation is consistent with the photodecarboxylation of the acid group. Similar results were obtained for other amino acids. The data were fitted to a first-order decay, as described in the experimental section, to yield the half-lives summarized in Table 1.

IR spectroscopy was used during warm-up to monitor the loss of various species. Once at room temperature, the residual, nonvolatile, organic film that remained was washed from the window with water and analyzed via high-precision liquid chromatography (HPLC) using the OPA/NAC fluorescent labeling protocol of Zhao \& Bada (1995) on a Hewlett Packard 1100 series HPLC with a Vydac C-18 $4.6 \times 250 \mathrm{~mm} 5 \mu \mathrm{m}$ resin analytical column. This HPLC separation procedure allowed us to distinguish between the D- and L-forms, permitting us to check for UV-induced racemization.

\section{RESULTS}

We investigated the UV destruction of glycine, $\alpha$-L-alanine, $\beta$-alanine, and $\mathrm{AIB}$ in solid $\mathrm{Ar}, \mathrm{N}_{2}$, and $\mathrm{H}_{2} \mathrm{O}$ at $12 \mathrm{~K}$ by monitoring the disappearance of the following amino acid functional groups by IR spectroscopy during photolysis: $\mathrm{OH}(3540$ $\left.3570 \mathrm{~cm}^{-1}\right), \mathrm{CH}_{3}\left(2940-3000\right.$ and $\left.1360-1460 \mathrm{~cm}^{-1}\right), \mathrm{CO}$ $\left(1760-1800 \mathrm{~cm}^{-1}\right), \mathrm{CN}\left(\sim 1110 \mathrm{~cm}^{-1}\right), \mathrm{C}-\mathrm{COOH}(\sim 780$ and $\left.805 \mathrm{~cm}^{-1}\right)$, and $\mathrm{C}-\mathrm{CH}_{3}\left(\sim 850 \mathrm{~cm}^{-1}\right)$.

Figure 1 shows the evolution of the IR spectrum of glycine isolated in $\mathrm{Ar}$ at $12 \mathrm{~K}$ as a function of UV photolysis time. During the 30 minute exposure to UV irradiation, all the absorption bands due to glycine diminish gradually and simultaneously. The growth of the dominant photoproduct, $\mathrm{CO}_{2}$, is strongly correlated with the decrease of the amino acid carbonyl $(\mathrm{C}=\mathrm{O})$ stretch (Fig. 2), consistent with the photodecarboxylation of the acid group from the amino acid. The loss of $\mathrm{CO}_{2}$ from glycine would presumably leave behind methylamine $\left(\mathrm{H}_{3} \mathrm{C}-\mathrm{NH}_{2}\right)$ as the other major product, but the expected peaks at 2970 and $2900 \mathrm{~cm}^{-1}$ are barely detectable, and no obvious $\mathrm{C}-\mathrm{N}$ single bond appears. One plausible scenario is that the methylamine rapidly decays to methylimine $\left(\mathrm{H}_{2} \mathrm{C}=\mathrm{NH}\right)$, which decomposes to yield $\mathrm{HCN}$. The presence of $\mathrm{HCN}$ is evident from the prominent peak near $3300 \mathrm{~cm}^{-1}$ and is consistent with 
TABLE 1

Amino Acid Half-Lives

\begin{tabular}{ccccc}
\hline \hline Matrix & $\begin{array}{c}\text { Half-Life } \\
(\mathrm{s})\end{array}$ & $\begin{array}{c}\text { Photon Dose } \\
\left(\times 10^{17} \mathrm{~cm}^{-2}\right)\end{array}$ & $\begin{array}{c}\text { Half-Life in DISM } \\
(\mathrm{yr})\end{array}$ & $\begin{array}{c}\text { Half-Life in DCs } \\
\left(\times 10^{6} \mathrm{yr}\right)\end{array}$ \\
\hline Glycine-Ar $\ldots \ldots \ldots$ & 1000 & 10 & 320 & 32 \\
Glycine- $\mathrm{H}_{2} \mathrm{O} \ldots \ldots$ & 1000 & 10 & 320 & 32 \\
Glycine- $\mathrm{N}_{2} \ldots \ldots \ldots$ & 700 & 7 & 220 & 22 \\
${ }^{(1)}$ Glycine- $\mathrm{N}_{2} \ldots \ldots$ & 1525 & 15 & 475 & 47 \\
Alanine-Ar $\ldots \ldots \ldots$ & 850 & 8.5 & 270 & 27 \\
Alanine- $\mathrm{H}_{2} \mathrm{O} \ldots \ldots$ & 800 & 8 & 250 & 25 \\
Alanine- $\mathrm{N}_{2} \ldots \ldots \ldots$ & 250 & 2.5 & 80 & 8 \\
AIB- $\mathrm{N}_{2} \ldots \ldots \ldots \ldots$ & 400 & 4 & 125 & 25 \\
AIB- $\mathrm{H}_{2} \mathrm{O} \ldots \ldots \ldots$ & 800 & 8 & 250 & 23 \\
$\beta$-alanine- $\mathrm{N}_{2} \ldots \ldots$ & 725 & 7.25 & 230 & 25 \\
\hline
\end{tabular}

Note. - This table lists the half-lives (in seconds) for all measured amino acids exposed to UV irradiation for different matrices and their corresponding lifetimes (in years) in the diffuse ISM (DISM, assuming a flux of $10^{8}$ photons $\mathrm{cm}^{-2} \mathrm{~s}^{-1}$; Mathis et al. 1983) and in dense clouds (DCs, assuming a cosmic-ray-induced UV flux of $10^{3}$ photons $\mathrm{cm}^{-2} \mathrm{~s}^{-1}$; Prasad \& Tarafdar 1983). The rates have been derived from the reduction of the CO stretch $\left(1780 \mathrm{~cm}^{-1}\right)$ of the acid group and the formation of the photoproduct $\mathrm{CO}_{2}\left(2340 \mathrm{~cm}^{-1}\right)$, respectively. In $\mathrm{H}_{2} \mathrm{O}$ matrices, we can only obtain indirect information about the stability of amino acids by measuring the photoproduct $\mathrm{CO}_{2}$. The superscript " (1)" indicates experiments with a $\mathrm{CaF}_{2}$ window, which allows only photons of less than $6 \mathrm{eV}$ to pass. Our half-lives measured in the laboratory provide a lower limit of amino acid destruction (see $\S 4$ ).

the smaller peaks at 2100 and $711 \mathrm{~cm}^{-1}$ (Moore, Pimentel, \& Goldfarb 1965). The methylimine intermediate is consistent with very weak peaks at $2940,1645,1360$, and $1070 \mathrm{~cm}^{-1}$ and a feature expected at $1115 \mathrm{~cm}^{-1}$, which is obscured by glycine peaks (Moore et al. 1965). Attributing the peaks at 1645 and $1070 \mathrm{~cm}^{-1}$ solely to the methylimine is complicated, however, by the presence of $\mathrm{NH}_{3}$, which also displays features in this region (Jacox \& Milligan 1963).

The UV photodestruction of the other amino acids results in very similar behavior (see Table 1). The rates of the photodestruction of glycine and $\beta$-alanine in $\mathrm{N}_{2}$ are estimated to be the same within experimental errors, whereas AIB and Lalanine in $\mathrm{N}_{2}$ were observed to decompose more rapidly. However, the rates of the photodestruction of glycine and L-alanine in the Ar matrix were very similar. We observed a somewhat faster decrease in the half-life of glycine and L-alanine in an $\mathrm{N}_{2}$ matrix compared with that in $\mathrm{Ar}$ or $\mathrm{H}_{2} \mathrm{O}$. The half-lives of L-alanine and AIB in $\mathrm{H}_{2} \mathrm{O}$ ice were identical (Table 1).

Blocking the $\mathrm{Ly} \alpha$ flux of the lamp with $\mathrm{a} \mathrm{CaF}_{2}$ window (which cuts out the wavelengths less than $150 \mathrm{~nm}$ ) only increased the half-life of glycine in $\mathrm{N}_{2}$ by a factor of 2 (Table 1). This is roughly proportional to the fraction of total photons filtered by the $\mathrm{CaF}_{2}$ (although the blocked photons are of much higher energy) and demonstrates that the lower energy $(\sim 160 \mathrm{~nm})$ photons are as efficient as Ly $\alpha$ in causing glycine decomposition.

The residual material left after the photolysis and warm-up of an $\mathrm{N}_{2}$ : L-alanine mixture was washed from the window using methanol. The remaining alanine was found, by HPLC, to have racemized at the $3 \%$ level. The racemization can be attributed entirely to photolysis since it was not observed in control experiments without UV irradiation. This has implications for maintaining an enantiomeric excess through environments where ionizing radiation is abundant (see below).

\section{DISCUSSION AND ASTROPHYSICAL IMPLICATIONS}

The photodestruction rate constants obtained in this study (Table 1) can be used to predict amino acid survival in different extraterrestrial environments. Our results show that amino acids have a limited stability against photolysis as free species and when embedded in solid surfaces that can be efficiently penetrated by UV photons. It is important to note, however, that these rate constants for photodecomposition in $\mathrm{Ar}$ (solid phase) are probably 5-10 times less than they would be if the amino acids were in the gas phase, because of the "cage effect" and because the solid matrix can scatter radiation and dissipate energy that might otherwise result in bond rupture (Andrews 1973).

These results show that UV photolysis induces the efficient destruction of amino acids and the formation of $\mathrm{CO}_{2}$ and $\mathrm{HCN}$ in the ice matrix. The destruction is independent of the size and structure for the amino acids studied and is fairly insensitive to the matrix. In all cases, the amino acids are almost completely decarboxylated after 30 minutes of exposure to $\sim 1 \times$ $10^{15}$ photons $\mathrm{cm}^{-2} \mathrm{~s}^{-1}$, corresponding to a total UV flux of $\sim 2 \times 10^{18}$ photons $\mathrm{cm}^{-2}$.

\subsection{Survival of Amino Acids in Interstellar Clouds}

In dense clouds, little of the UV flux from stars penetrates the high column density of dust and gas, and only the cosmicray-induced UV flux of $\sim 10^{3}$ photons $\mathrm{cm}^{-2} \mathrm{~s}^{-1}$ is relevant for the photochemistry in such environments (Prasad \& Tarafdar 1983). Our Ar matrix results suggest that even in these quiescent regions, a significant fraction of gas-phase amino acids should be destroyed within the lifetime of a typical cloud $\left(10^{7} \mathrm{yr}\right)$; our half-lives measured in the laboratory provide a lower limit of amino acid destruction.

In contrast to dense clouds, the UV flux in the diffuse ISM is much higher $\left(\sim 10^{8}\right.$ photons $\mathrm{cm}^{-2} \mathrm{~s}^{-1}$ for photons greater than $6 \mathrm{eV}$; Mathis, Mezger, \& Panagia 1983). Thus, the timescale for the survival of amino acids in the diffuse gas is at most a few hundred years. Similarly, in star formation regions, like Orion, there is more UV than in dense clouds, and so the survival of amino acids is limited to short timescales. Even in such energetic environments, amino acids could potentially survive longer if protected within interstellar grains.

Our studies of amino acid destruction in solid $\mathrm{H}_{2} \mathrm{O}$ at $12 \mathrm{~K}$ suggest that in ice mantles of interstellar grains, amino acids may have a slightly longer lifetime, but not significantly if they are thin enough to be penetrated by UV. This rapid photodecomposition may explain why amino acids have been so hard to detect in the past in the gas phase. If, however, amino acids are formed in the ice (i.e., by energetic processing; Briggs et 
al. 1992; M. P. Bernstein, J. P. Dworkin, S. A. Sandford, \& L. J. Allamandola 2001, in preparation), then they may be present at low levels, depending on the steady state equilibrium between formation and destruction.

In the lab, the molecule $\mathrm{HCOOH}$, a direct tracer of grain evaporation, shows a photodestruction rate similar to that of glycine (Ehrenfreund et al. 2001) and is present in the interstellar gas in low abundance $\left(\mathrm{HCOOH} / \mathrm{H}_{2} \sim 1 \times 10^{-10}\right)$. In interstellar grains, $\mathrm{HCOOH}$ is tentatively detected with an abundance of $\sim 3 \%$ relative to $\mathrm{H}_{2} \mathrm{O}$ ice (Whittet et al. 1996), corresponding to $\mathrm{HCOOH} / \mathrm{H}_{2}<10^{-6}$. The differences between solid-state and gas-phase abundances of $\mathrm{HCOOH}$ may indicate an efficient destruction mechanism and/or subsequent reaction pathways of $\mathrm{HCOOH}$ in the gas phase to larger molecules (Ehrenfreund et al. 2001). Although $\mathrm{HCOOH}$ is simpler and probably formed more efficiently, inasmuch as it is analogous to glycine, its presence suggests that the detection of glycine in low-UV interstellar regions should not be excluded.

\subsection{Survival of Amino Acids in the Solar System}

As in the ISM, the survival of amino acids in exposed environments in a planetary system is problematic. The UV flux from the Sun comes mainly from chromospheric lines and continuum emission. A significant fraction of the UV photons are associated with the Ly $\alpha$ lines $\left[(3-5) \times 10^{11}\right.$ photons $\mathrm{cm}^{-2} \mathrm{~s}^{-1}$, at $1 \mathrm{AU}$. The solar flux above $6 \mathrm{eV}$ (of $3 \times 10^{13}$ photons $\mathrm{cm}^{-2} \mathrm{~s}^{-1}$ ) is sufficient to destroy all gas-phase amino acids that are free or amino acids on the surface of grains in a few hours at 1 AU. Scaling the solar constant to 5 AU, amino acids on the very surface of Europa would be destroyed in a few days.

UV measurements of T Tauri stars resembling the young Sun obtained with IUE indicate that such stars emit up to $10^{4}$ times more UV than the present Sun (Canuto et al. 1982). Thus, the rate of destruction of amino acids may have been greater in the past, although these ratios reflect transition region line emission and not the vacuum UV integrated below $200 \mathrm{~nm}$ (B. H. Foing 2000, private communication). Models of protoplanetary disks suggest a multilayer structure (Aikawa \& Herbst 1999) in which amino acids would not survive in the upper atmosphere of disks exposed to solar and interstellar radiation but may endure in the very dense $\left(10^{8}-10^{9} \mathrm{~g} \mathrm{~cm}^{-3}\right)$ midplane region where UV photons are absorbed.

For glycine, an upper limit of less than 0.005 relative to $\mathrm{H}_{2} \mathrm{O}$ has been established in the coma of Hale-Bopp (Crovisier et al. 1999). While it may be that amino acids remain undetected in cometary comae (and in the ISM) simply because they are not present, perhaps they persist in the ice but are destroyed rapidly in the gas phase before they can accumulate there. Our measurements are consistent with this latter scenario, in which glycine may never be observed in the comae of comets, but the amino acids in meteorites might still be the direct result of interstellar chemistry (as opposed to an aqueous alteration). However, the incorporation of interstellar amino acids into solar system bodies must proceed in a manner that keeps them protected from UV radiation at all times.

Recent observations of nonracemic amino acids in carbonaceous chondrites (Cronin \& Pizzarello 1997) have renewed interest in the preferential creation or destruction of amino acids of one handedness over another. Our experiments indicate that L-alanine was only a few percent racemized after 30 minutes. So UV photolysis should pose a minor obstacle to maintaining an enantiomeric excess since the rate of destruction is much higher than the rate of racemization.

\section{CONCLUSION}

Amino acids are highly susceptible to UV photodestruction, even under exposure to UV photons of relatively low energy. Their limited photostability suggests that amino acids have not been detected because they are destroyed before they can accumulate in the gas phase. Although amino acids may be detected by radio observations in UV-shielded environments (such as hot cores), merely traces will survive in any environment with an elevated UV flux. This applies to both interstellar and solar system environments, at least out to distances of $5 \mathrm{AU}$, and would appear to be inconsistent with recent studies of the survival of amino acids in Earth orbit (Barbier et al. 1998).

Our results place constraints on the inventory of amino acids for exogenous delivery to the early Earth and thus on prebiotic evolution. However, this does not preclude the amino acids in meteorites having formed in the ISM. It simply requires that amino acids be shielded from UV in protected environments, such as in dust grains and meteorites and in the interiors of small bodies, i.e., comets and asteroids prior to delivery to early planets.

We are grateful to Bob Walker for technical support and W. F. Thi for computer support. We thank S. B. Charnley and O. Botta for their discussions. P. E. was supported by the Netherlands Research School for Astronomy (NOVA). The Ames Astrochemistry lab was supported by NASA's Origins of Solar Systems (344-37-00-07), by NASA Astrobiology (34450-92-02), and by the NASA Ames Director's Discretionary Fund. We thank Art Weber for technical assistance.

\section{REFERENCES}

Aikawa, Y., \& Herbst, E. 1999, A\&A, 351, 233

Andrews, L. 1973, in Vibrational Spectroscopy of Trapped Species, ed. H. E. Hallam (London: Wiley), 179

Barbier, B., Chabin, A., Chaput, D., \& Brack, A. 1998, Planet. Space Sci., 46/ 4, 391

Bernstein, M. P., Sandford, S. A., \& Allamandola, L. J. 1999, Sci. Am., 281(1), 42

Blank, J. G., Miller, G. H., \& Winans, R. E. 2001, Origins Life Evol. Biosphere, in press

Briggs, R., et al. 1992, Origins Life Evol. Biosphere, 22, 287

Canuto, V. M., Levine, J. S., Augustsson, T. R., \& Imhoff, C. L. 1982, Nature, 296, 816

Ceccarelli, C., Loinard, L., Castets, A., Faure, A., \& Lefloch, B. 2000, A\&A, 362,1122
Charnley, S. B., Ehrenfreund, P., \& Kuan, Y. 2001, Spectrochim. Acta, in press

Chyba, C. F., Thomas, P. J., Brookshaw, L., \& Sagan, C. 1990, Science, 249, 366

Cronin, J. R., \& Chang, S. 1993, The Chemistry of Life's Origin, ed. J. M. Greenberg et al. (Dordrecht: Kluwer), 209

Cronin, J. R., \& Pizzarello, S. 1997, Science, 275, 951

Crovisier, J., Bockelee-Morvan, D., Colom, P., Biver, N., Despois, D., Lis, D., Benford, D. J., \& Mehringer, D. 1999, AAS DPS Meeting, 31, 32.02 Ehrenfreund, P., \& Charnley, S. B. 2000, ARA\&A, 38, 427

Ehrenfreund, P., d'Hendecourt, L., Charnley, S. B., \& Ruiterkamp, R. 2001, J. Geophys. Res., in press

Gerakines, P. A., Moore, M. H., \& Hudson, R. L. 2000, A\&A, 357, 793

Hudgins, D. M., \& Allamandola, L. J. 1995, J. Phys. Chem., 99, 3033

Irvine, W. M. 1998, Orig. Life Evol. Biosphere, 28, 365 
Jacox, G., \& Milligan, D. E. 1963, Spectrochim. Acta, 19, 1173

Mathis, J. S., Mezger, P. G., \& Panagia, N. 1983, A\&A, 128, 212

Moore, C. B., Pimentel, C. G., \& Goldfarb, T. D. 1965, J. Chem. Phys., 43, 63

Oró, J. 1961, Nature, 190, 389

Pierazzo, E., \& Chyba, C. F. 1999, Meteoritics \& Planet. Sci., 34(6), 909

Pizzarello, S., Krishnamurthy, R. V., Epstein, S., \& Cronin, J. R. 1991, Geochim. Cosmochim. Acta, 55, 905
Prasad, S. S., \& Tarafdar, S. P. 1983, ApJ, 267, 603

Sandford, S. A. 1996, Meteoritics, 31, 449

Snyder, L. 1997, Orig. Life Evol. Biosphere, 27, 115

Warneck, P. 1962, Appl. Opt., 1, 721

Wexler, A. S. 1967, Appl. Spectrosc. Rev., 1, 29

Whittet, D. C. B., et al. 1996, A\&A, 315, L357

Zhao, M., \& Bada, J. L. 1995, J. Chromatogr. A, 690, 55 